\title{
The RICH detector of the NA62 experiment at CERN
}

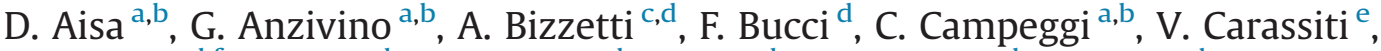

A. Cassese ${ }^{\mathrm{d}, \mathrm{f}}$, P. Cenci $^{\mathrm{b}}$, R. Ciaranfi $^{\mathrm{d}}$, V. Duk ${ }^{\mathrm{b}}$, L. Farnesini ${ }^{\mathrm{b}}$, J.R. Fry ${ }^{\text {g,h }}$, E. Iacopini ${ }^{\mathrm{d}, \mathrm{f}}$, S. Lami ${ }^{\mathrm{i}}$, M. Lenti ${ }^{\mathrm{d}}$, F. Maletta ${ }^{\mathrm{d}}$, M. Pepe ${ }^{\mathrm{b}}$, R. Piandani ${ }^{\mathrm{i}}$, M. Piccini ${ }^{\mathrm{b}}$, A. Piluso ${ }^{\mathrm{a}, \mathrm{b}}$,

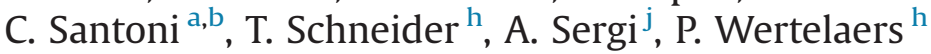

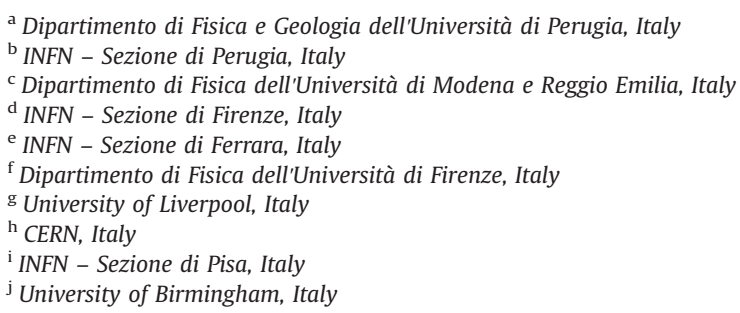

\section{A R T I C L E I N F O}

Available online 23 July 2014

Keywords:

Cherenkov radiation

$\mathrm{RICH}$

Kaon physics

Rare decays

\begin{abstract}
A B S T R A C T
The NA62 experiment at CERN aims to measure the branching ratio of the ultra-rare charged kaon decay $\mathrm{K}^{+} \rightarrow \pi^{+} \nu \bar{\nu}$ with a $10 \%$ accuracy and with a background contamination at the $10 \%$ level. Since the branching ratio of this decay is $O\left(10^{-10}\right)$, to fulfill such request one of the main backgrounds, the decay $\mathrm{K}^{+} \rightarrow \mu^{+} \nu\left(\mathrm{BR} \sim 63 \%\right.$ ), must be suppressed by a rejection factor of $4 \times 10^{-13}$ (assuming $10 \%$ signal acceptance). This can be partially accomplished using a combination of kinematical cuts $\left(8 \times 10^{-6}\right)$ and the different power of penetration through matter of pions and muons $\left(10^{-5}\right)$. A further $5 \times 10^{-3}$ suppression factor will be provided by a RICH detector, in a momentum range between 15 and $35 \mathrm{GeV} / \mathrm{c}$. The details of the RICH project as well as the results from test runs performed on a RICH prototype of the same length of the final detector will be presented. The current status of the construction and the description of the final readout and trigger electronics will also be reviewed.
\end{abstract}

(c) 2015 CERN for the benefit of the Authors. Published by Elsevier B.V. This is an open access article under the CC BY license (http://creativecommons.org/licenses/by/4.0/).

\section{Introduction}

The NA62 experiment [1] at CERN will start to take data in fall 2014 to measure the branching ratio of the ultra-rare charged kaon decay $\mathrm{K}^{+} \rightarrow \pi^{+} \nu \bar{\nu}$ with a $10 \%$ accuracy and with a background contamination at the $10 \%$ level. The downstream RICH detector will be fundamental to suppress the background from decays with a muon in the final state (mainly $\mathrm{K}^{+} \rightarrow \mu^{+} \nu$ ). In fact the RICH will allow to reject muons contaminating the pion sample with a suppression factor of the order of $5 \times 10^{-3}$ in a momentum range between 15 and $35 \mathrm{GeV} / c$. The RICH detector must also provide the pion crossing time with a resolution of the order of $100 \mathrm{ps}$ to minimize wrong matching with the decaying kaon measured by an upstream detector. The RICH will stand a rate of about $10 \mathrm{MHz}$ and will be a key element of the NA62 trigger system.

\section{The NA62 RICH}

The NA62 RICH detector (see Fig. 1) consists of a cylindrical vacuum-proof steel vessel, about $18 \mathrm{~m}$ long and with diameter ranging from 3.4 to $4 \mathrm{~m}$ (four sections). Neon gas at atmospheric pressure and room temperature is used as radiator element (refractive index $n=1.0000665$ for a photon wavelength of $\lambda=420 \mathrm{~nm}$ ). An evacuated vacuum tube (beam pipe) is present along all the detector axis to avoid the interaction of the particles of the charged beam with the radiator. The Cherenkov light is reflected by a mosaic of 18 hexagonal and 2 semi-hexagonal (central area) spherical mirrors with $17 \mathrm{~m}$ focal length, made of $2.5 \mathrm{~cm}$ thick glass. They cover a total surface of about $3 \mathrm{~m}$ in diameter, providing the full coverage for the Cherenkov photons emitted by $\pi$ and $\mu$ traveling inside the geometrical acceptance of the downstream detectors. The right and the left sides of the mirror surfaces have two different center of curvatures in order to avoid that the reflected photons hit the beam pipe without reaching the sensitive devices. The mirrors will be individually hung on a light aluminium structure (support panel) and moved by means of two piezo-motors each, in order to align the light toward the two focal points.

The reflected light is collected by about 2000 Hamamatsu R7400-U03 photomultipliers (PM) with $18 \mathrm{~mm}$ pixel size and good 


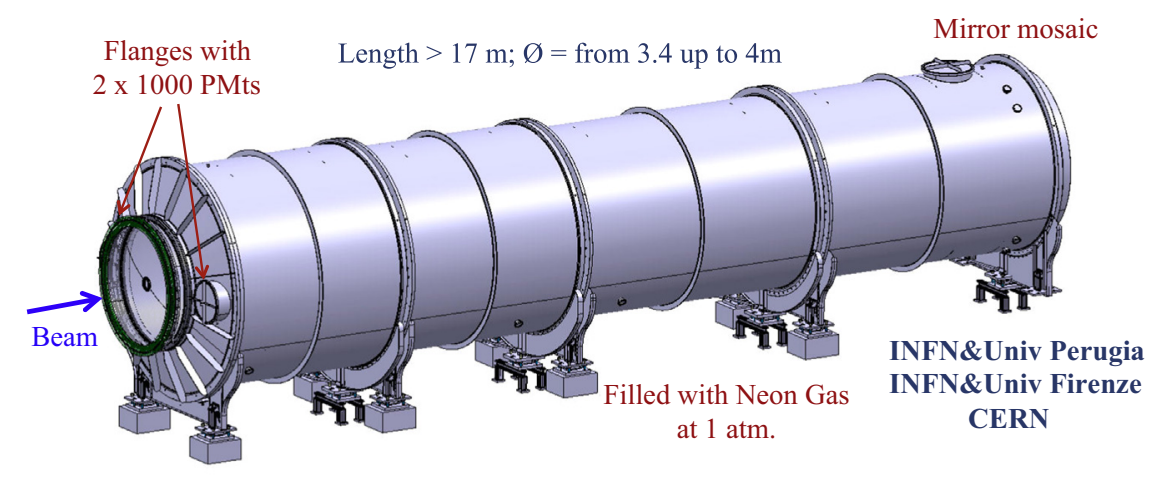

Fig. 1. Layout of the RICH vessel.

quantum efficiency and timing performances. These metal packaged PMs were chosen for their compactness and fastness after the comparison tests carried-on during the 2007 test run. The R7400 PM has a polyoxymethylene insulation cover of roughly cylindrical shape. The photocathode (with $8 \mathrm{~mm}$ minimum active diameter) is bialkali made and has a typical radiant sensitivity of $62 \mathrm{~mA} / \mathrm{W}$ at the $420 \mathrm{~nm}$ peak wavelength, corresponding to a $20 \%$ quantum efficiency; the PM wavelength sensitivity is between 185 and $650 \mathrm{~nm}$. The typical PM gain is $1.5 \times 10^{6}$ at $900 \mathrm{~V}$ (working voltage). The R7400-U03 typical rise time is $0.78 \mathrm{~ns}$, the transit time is $5.4 \mathrm{~ns}$ and the transit time jitter is $0.28 \mathrm{~ns}$ (FWHM). The eight PM dynode voltages are provided through a custom made HV divider (28 M $\Omega$ total resistance), which has a cylindrical shape and three cables: one for signal output and two cables for high voltage (negative) supply and for grounding (see Fig. 2).

The PMs are assembled in a compact hexagonal packing into two aluminium discs, placed on both the sides of the vessel at the entrance window in correspondence to the two different focal surfaces. Winston cones [2] carved in the discs and covered with aluminized mylar are used to convey the light onto the active PM area, thus increasing light collection.

The PMs will be powered by four CAEN SY2527 crates equipped with A1535S boards, each one providing $24 \mathrm{HV}$ channels. In order to reduce the number of modules, each HV channel will power 4 PMs. Quartz windows are used to separate the PMs from the Neon in order to avoid electrical discharges at the working voltage.

The PM signal is sent to custom-made current amplifiers with differential output and then to NINO chips [3] used as discriminators operating in time-over-threshold mode. The RICH readout and trigger electronics will make use of the integrated trigger and data acquisition system (TDAQ) developed to read most of the NA62 detector in a common way. The key element of the TDAQ system is an upgraded version of the LHC TELL1 board [4], called TEL62 [5] (see Fig. 3). Digitized signals from the RICH are sent to a custom-made TDC board [6] (TDCB) housing four CERN HPTDCs [7]. The TDCB (mezzanine board) sends leading and trailing time of the incoming signals to the TEL62 (mother board). In the TEL62 the signal is handled both to produce L0 trigger primitives and to send relevant data to the next element of the readout chain (online PC farm).

In the NA62 experiment a three-level trigger system will reduce the $10 \mathrm{MHz}$ detector rate to about $10 \mathrm{kHz}$. The Level 0 (L0) will decrease the event rate from 10 to $1 \mathrm{MHz}$ employing signals from RICH, photon veto system, charged hodoscope and muon veto detector. The higher level trigger is based on PCs and will reduce the rate of the events to $10 \mathrm{kHz}$ level. The excellent time resolution of the RICH can be exploited in the L0 trigger to determine the reference time of the tracks and this is a crucial feature to get an efficiency better than 95\% for signal events. Studies on multiplicity variables to identify multiple track events

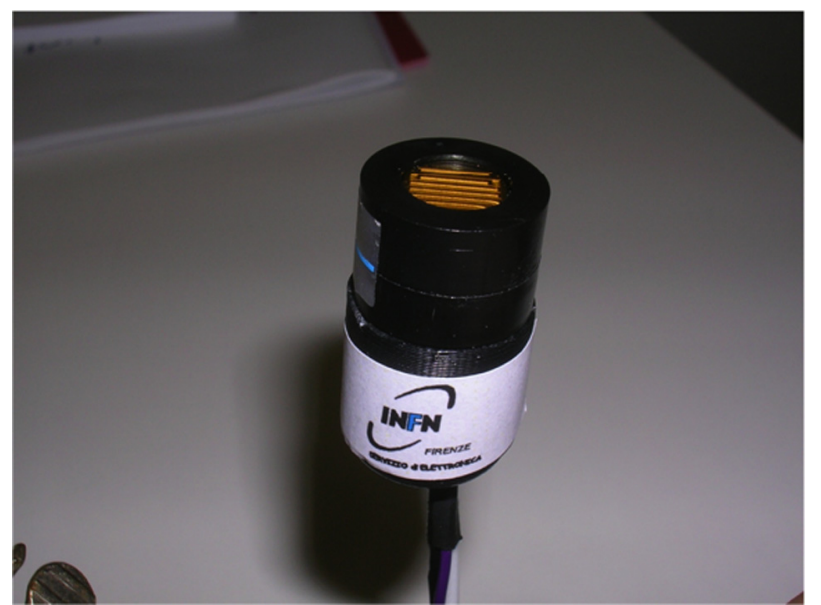

Fig. 2. The HAMAMATSU R7400 U-03 photomultiplier connected to the custom made divider.

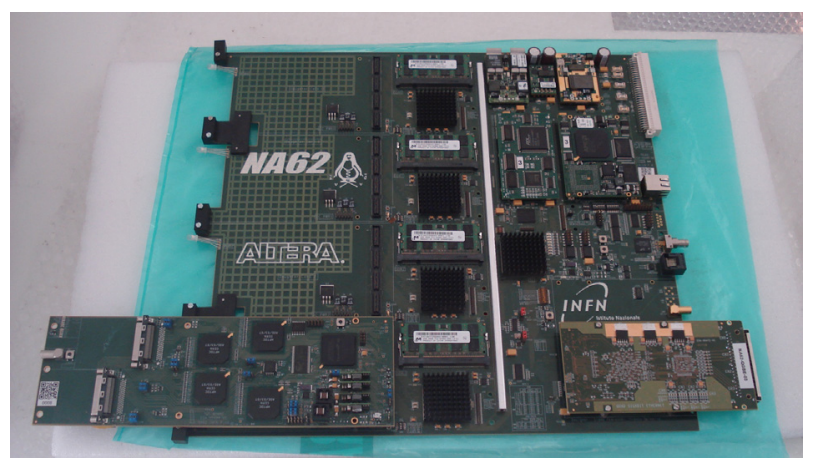

Fig. 3. The TEL62 mother board. At the bottom-left a plugged TDCB is also shown.

and to reject fake signals produced by electronics noise show promising results already at $\mathrm{L} 0$. At the next trigger level (L1) the RICH will provide the number and the position of the Cherenkov rings, helping to reject events with more than one charged particle in the final state. Charged particle with $\beta=1$ (electrons) can be even rejected using the reconstructed ring radius. The possibility to use a GPU-based system instead of standard CPUs, in order to speed up the reconstruction already at trigger level, is also under consideration [8]. In the last trigger level (L2) for each event the RICH data are merged with the data incoming from the other subdetectors; at this level complex selection criteria will be applied exploiting those combined information and the $\mathrm{RICH}$ will be fundamental to perform particle identification. 


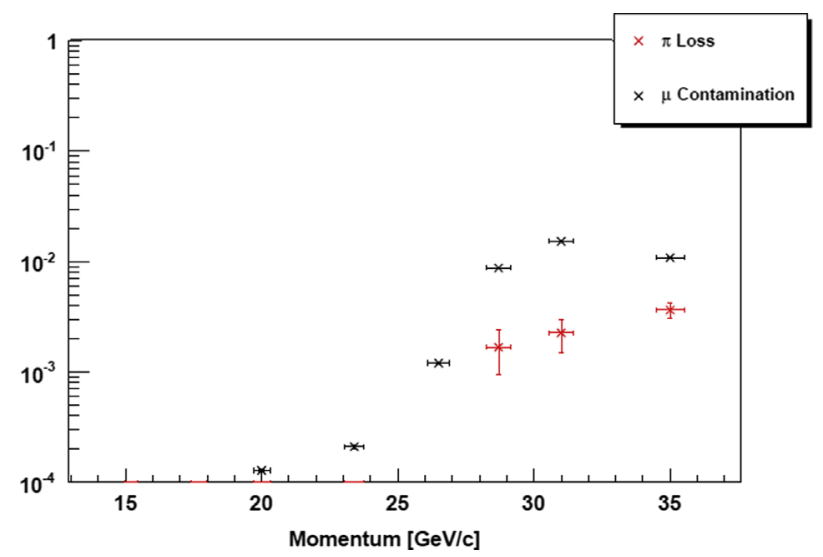

Fig. 4. $\mu$ mis-identification probability and inefficiency on $\pi$ selection as a function of pion momentum.

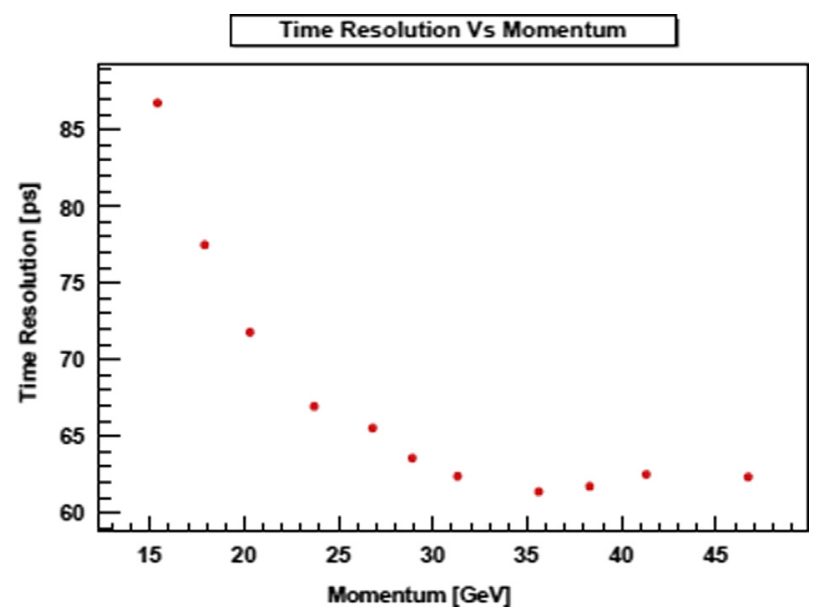

Fig. 5. Time resolution as a function of pion momentum, defined as the average root mean square of the selected hit times with respect to their average.

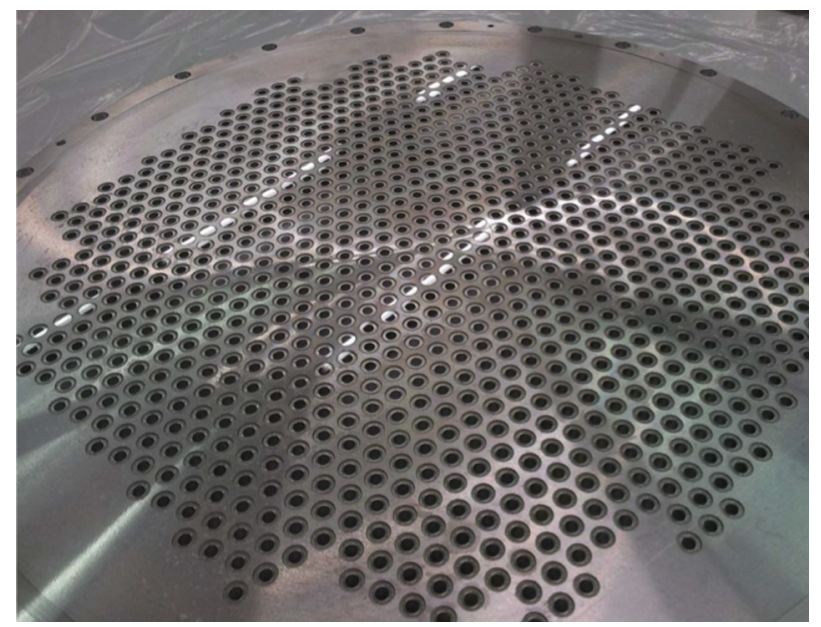

Fig. 6. One of the two flanges housing the photomultipliers of the RICH detector (quartz window side).

\section{Results from tests}

The detector performances have been validated in two tests of a full $17 \mathrm{~m}$ length prototype done at CERN with charged beams in 2007 and 2009. The 2007 test was useful to select the

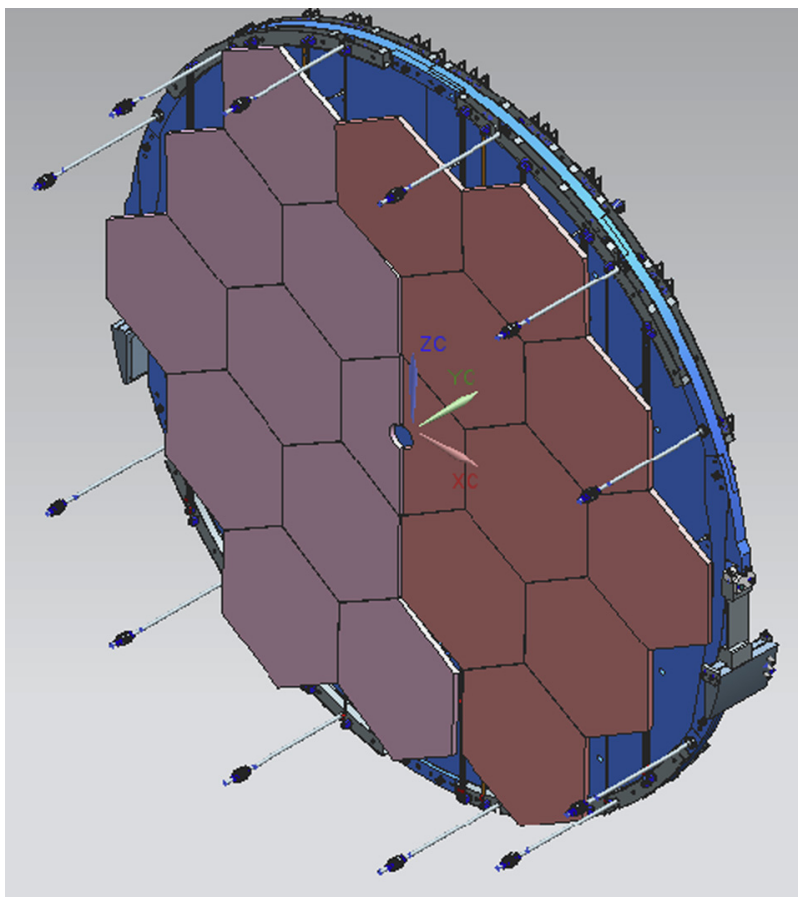

Fig. 7. Layout of the panel supporting the RICH mirrors.

photomultiplier type to be used for the final detector and has confirmed that a resolution better than 100 ps is achievable offline for the time measurement of charged particles [9]. With the data collected during the 2009 test has been shown that a pion sample can be selected with a muon contamination of $0.7 \%$ [10] (see Fig. 4); it has been obtained once the results of the test at different energies were properly weighted with the expected spectrum of the pions produced in the $\mathrm{K}^{+} \rightarrow \pi^{+} \sqrt{\nu}$ decay. The data analysis has also shown that a significant contribution to the measured contamination is due to $\delta$-electrons produced in the material in front of the RICH prototype. The $\pi-\mu$ separation can be improved up to the requested value either increasing the corresponding $\pi$ loss of about $1 \%$ or reducing the amount of material upstream the final RICH detector. The analysis of the 2009 data has also confirmed that a resolution better than $100 \mathrm{ps}$ is achievable for the time measurement of charged particles with momentum between 15 and $35 \mathrm{GeV} / \mathrm{c}$ (see Fig. 5).

\section{Status of the construction}

The construction of the detector is almost completed and the installation has started at the beginning of 2014; the RICH is on schedule to be ready for the first physics run foreseen in fall 2014. In particular, the 4 sections composing the vacuum-proof vessel, built in 2013, are in the process of being installed in the NA62 experimental area.

The two flanges housing the 2000 PMs were produced in 2012 and all the quartz windows have been already glued at CERN (see Fig. 6). The installation of the PMs in the flanges has already started in Firenze and the two parts will be delivered to CERN during the next summer.

The panel to support the mirrors (see Fig. 7) is under construction and will be delivered to CERN in spring 2014. The support panel will be made in aluminium honeycomb, in order to minimize the probability of particle interactions in front of the NA62 Electromagnetic Calorimeter that is located downstream with respect to the beam direction. A prototype of the support panel 
housing 3 dummy mirrors is under construction in Perugia and it is used to establish a correct procedure for the mirrors installation. The prototype is also used to design and produce the proper tools for the mirrors installation at CERN.

The 20 mirrors reflecting the Cherenkov light on the PM flanges have been already delivered to CERN and the aluminization and coating processes are just started.

\section{References}

[1] G. Anelli, et al., CERN-SPSC-2005-013 and CERN-SPSC-P-326, 2005.

[2] R. Winston, Journal of the Optical Society of America 60 (1970) 245.

[3] F. Anghinolfi, P. Jarron, A.N. Martemiyanov, E. Usenko, H. Wenninger, M.C. S. Williams, A. Zichichi, Nuclear Instruments and Methods in Physics Research Section A 533 (2004) 183.
[4] G. Haefeli, A. Bay, A. Gong, H. Gong, M. Muecke, N. Neufeld, O. Schneider, Nuclear Instruments and Methods in Physics Research Section A 560 (2006) 494.

[5] B. Angelucci, E. Pedreschi, M. Sozzi, F. Spinella, Proceedings of topical workshop on electronics for particle physics 2011 (TWEPP-11), Journal of Instrumentation 7 (2012) C02046.

[6] G. Collazuol, et al., in: Proceedings of the Nuclear Science Symposium Conference Record (NSS/MIC), IEEE, 2009, p. 1138.

[7] M. Mota, J. Christiansen, in: Proceedings of the 2000 Nuclear Science Symposium and Medical Imaging Conference Lyon, vol. 1520, 2000.

[8] G. Lamanna, in: Proceedings of the 8th International Workshop on Ring Imaging Cherenkov Detectors (RICH 2013) 766 (2014) 241.

[9] G. Anzivino, et al., Nuclear Instruments and Methods in Physics Research Section A 593 (2008) 314.

[10] B. Angelucci, et al., Nuclear Instruments and Methods in Physics Research Section A 621 (2010) 205. 